Dermatology 2019;235:522-524

DOI: $10.1159 / 000501590$

\section{Tattoo Reactions Associated with Targeted Therapies and Immune Checkpoint Inhibitors for Advanced Cancers: A Brief Review}

\author{
Nicolas Kluger ${ }^{a, b}$ \\ a Departments of Dermatology, Allergology and Venereology, \\ Helsinki University Central Hospital, Helsinki, Finland; \\ b"Tattoo" Consultation, Department of Dermatology, \\ Bichat-Claude Bernard Hospital, Assistance Publique - \\ Hôpitaux de Paris, Paris, France
}

\section{Keywords}

Immune checkpoint inhibitor · Granuloma .

Molecular targeted therapies · Sarcoidosis · Tattooing

\section{Introduction}

Cutaneous reactions on permanent tattoos have been reported in the past during immune restauration syndrome under highly active antiretroviral therapy for human immunodeficiency virus $[1,2]$ as well as during interferon therapy for hepatitis $C$ with the induction of sarcoidosis [3, 4]. Besides, anecdotal cases of granulomatous tattoo reaction under tumor necrosis factor $\alpha$ inhibitors have been reported [5]. Inhibitors of the mitogen-activated protein (MAP) kinase pathway and immune checkpoint inhibitors (ICPIs) are widely used now in the management of advanced malignancies. However, ICPIs are associated with a wide range of dermatologic manifestations including sarcoidosis [6] and granulomatous reactions [7]. With the increased popularity of tattoos worldwide, cases of tattoo reactions are slowly increasing. We reviewed here all the case reports of tattoo reactions associated with targeted therapies and ICPIs.

\section{Materials and Methods}

We performed a review of the existing English- and Frenchlanguage literature on patients who developed a reaction on $\geq 1$ tattoo during or after targeted therapies and ICPIs. The databases MEDLINE (1946-2019) and Scopus (1823-2019) were initially searched on June 6,2019 . The following keywords were used: tattoo, tattoos, tattooing, molecular targeted therapies, BRAF inhibitor; MEK inhibitor; immune checkpoint inhibitor; as well as the following molecules: avelumab, nivolumab, pembrolizumab; ipilimumab; dabrafenib, encorafenib, vemurafenib; binimetinib, cobimetinib, trametinib. All the articles reporting on $\geq 1$ case(s) of targeted therapies/ICPI-associated tattoo reactions were included. We also included conference abstracts if relevant.

\section{Results}

To the best of our knowledge, 10 cases ( 6 men, 4 women; mean age 44.5 years, range $22-59$ ) have been reported since 2012 . The main characteristics are summarized in Table 1. Six patients received a combination of BRAF inhibitor (BRAFi) associated with a MEK inhibitor (MEKi), 2 patients a PD-1/PD-L1 inhibitor (PD-1i/ PD-L1i) and 2 patients a CTLA-4 inhibitor (CTLA-4i), including one in combination with a PD-1i. We found that the clinical presentation was different according to culprit drugs. Tattoo eruption associated with BRAFi + MEKi presented mostly as a complete infiltration/induration of dark-colored tattoos with striking inflammatory borders in 5 out of 6 cases $[10-13,16]$. Tattoo reactions with CTLA-4i or PD-1i/PD-L1i were reminiscent of what is usually observed with sarcoidosis or granulomatous tattoo reactions in general, e.g. papules and nodules scattered within black tattoos $[8,9$, $14,15]$. Tattoos were dark/black in $86 \%(6 / 7)$ of the cases. In case of multicolored tattoos, other colors were spared. Delays of onset after the first cycle were as follows from the earliest to the latest: CTLA-4i + PD-1i (2 months) > BRAFi + MEKi (2-6 months) > PD-1i/PD-L1i (9-15 months). Only 1 patient had additional cutaneous sarcoids on plain skin and erythema nodosum [9]. Biopsies showed noncaseating granulomas in $67 \%(6 / 9)$, including all the cases with CTLA-4i and/or PD-1i/PD-L1i. In the 3 remaining cases, there was only a lymphocytic infiltration without any granulomas $[10,12,13]$. Angiotensin-converting enzyme was elevated in 2 cases of granulomatous reactions $[9,15]$. Lung involvement and lymph nodes were reported in 60\% (6/10). Management was highly variable according to authors. It included local corticosteroids, combination of local and systemic corticosteroids, abstention with either maintenance or discontinuation of the treatment, and switch to $\mathrm{PD}$-1i or surgery. One patient experienced relapses with BRAFi + MEKi cycles [10]. Overall, outcome was always favorable.

\section{Discussion}

Granulomatous eruptions under ICPIs are an uncommon immune-related adverse event that can become more and more frequently encountered with the increased use of this therapeutic class [7]. All the patients under PD-1i/PD-L1i/CTLA-4i developed sarcoid-like reactions on tattoos. Delays of onset and outcomes were here in line with what is known from the literature [7]. On the other hand, cutaneous granulomatous reactions and sarcoidosis are rather infrequent with BRAFi [17]. Out of the 5 patients under BRAFi + MEKi with available biopsies, 3 displayed a lymphocytic infiltration in the dermis without granulomas. Besides, the clinical presentation was rather different with a marked erythema around the tattoos which is totally unusual compared to the usual tattoo granulomatous reactions we often see in practice [4, $18,19]$. Only 1 patient did not present such an inflammatory reaction around the affected tattoos [16]. To date, there is no case report of tattoo reaction with BRAFi alone. In 1 patient [11], the reaction developed 3 months after MEKi introduction. The differ-

\section{KARGER}

(c) 2019 S. Karger AG, Basel 


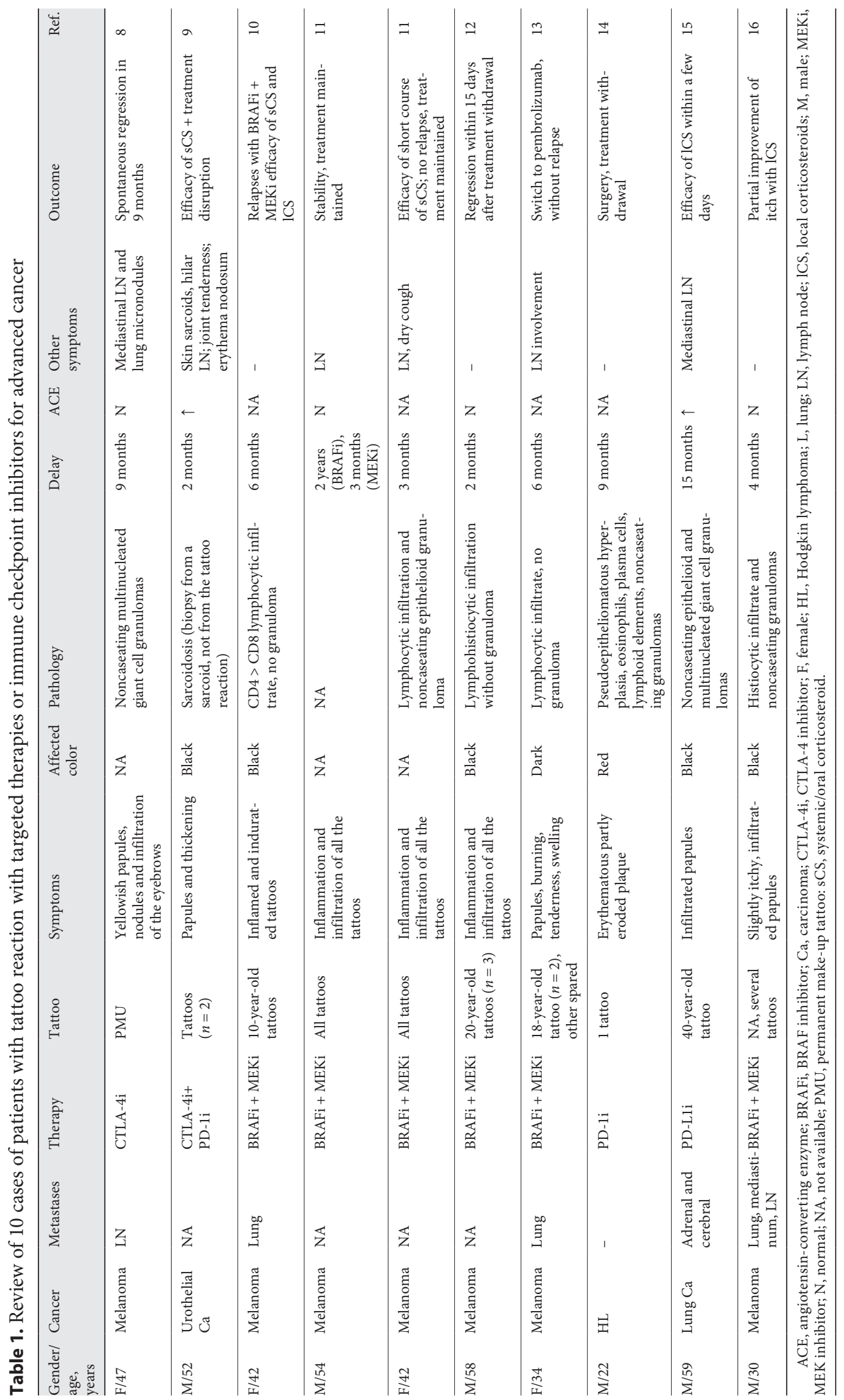


ences observed in histology may be related to sampling, and repeated biopsies could disclose genuine granulomas. Another hypothesis is that those reactions belong to different parts of the same spectrum of cutaneous immune-related adverse events.

The physiopathogeny of granuloma formation under ICPIs may be related to the activation of the innate immune system, the dysfunction of regulatory T cells and the expansion of Th7 cells. Reduction of CTLA-4 expression may contribute to increased proliferation capacity [7]. On the other side, the inhibition of BRAF and MEK could lead to a paradoxical activation of ERK as reported recently [20]. Besides, the inhibition of MEK, which is a negative regulator of AKT [21], could lead to a possible activation of the $\mathrm{AKT} / \mathrm{mTOR}$ pathway, which could induce the formation of granulomas [22]. This review is limited by the low number of cases and the quality of the reports, sometimes originating from posters.

With the increased prevalence of tattoos among the population, tattoo reactions associated with targeted therapies and immunotherapies for cancer may become more frequent. Those reactions affect mostly old black tattoos. We cannot rule out that the seeming affinity of those reactions to black tattoos is fortuitous. Histologically reactions range from lymphocytic infiltration to granulomatous reaction. They may be associated with genuine systemic sarcoidosis that should not be mistaken for metastatic progression [7]. Close monitoring is mandatory, invasive biopsies may be necessary if in doubt. Outcome is usually favorable, and treatment discontinuation is not obligatory.

Key Message

The use of immune checkpoint inhibitors and targeted therapies for advanced cancers may lead to granulomatous reactions and sarcoidosis within tattoos.

\section{Disclosure Statement}

The authors declare no conflict of interest.

\section{References}

1 Silvestre JF, Albares MP, Ramón R, Botella R. Cutaneous intolerance to tattoos in a patient with human immunodeficiency virus: a manifestation of the immune restoration syndrome. Arch Dermatol. 2001 May;137(5): $669-70$.

2 Gamba CS, Lambert Smith F, Wisell J, Brown M. Tattoo reactions in an HIV patient: autoeczematization and progressive allergic reaction to red ink after antiretroviral therapy initiation. JAAD Case Rep. 2015 Nov; 1(6):395-8.

3 Perera GK, Calonje E. Systemic sarcoidosis presenting in a tattooed man undergoing treatment for hepatitis C. Clin Exp Dermatol. 2006 May; 31(3):387-9.

4 Kluger N. Sarcoidosis on tattoos: a review of the literature from 1939 to 2011. Sarcoidosis Vasc Diffuse Lung Dis. 2013 Aug;30(2):86-102.

5 Bachmeyer C, Blum L, Petitjean B, Kemiche F, Pertuiset E. Granulomatous tattoo reaction in a patient treated with etanercept. J Eur Acad Dermatol Venereol. 2007 Apr;21(4):550-2.
6 Sibaud V. Dermatologic Reactions to Immune Checkpoint Inhibitors: Skin Toxicities and Immunotherapy. Am J Clin Dermatol. 2018 Jun; 19(3):345-61.

7 Cornejo CM, Haun P, English J 3rd, Rosenbach M. Immune checkpoint inhibitors and the development of granulomatous reactions. J Am Acad Dermatol. 2018 Aug 6. pii: S0190-9622(18)32355-7. doi: https://doi. org/10.1016/j.jaad.2018.07.051. Epub ahead of print.

8 Mallet S, Gaudy-Marquette C, Monestier S, Beaussault Y, Grob JJ, Richard MA. Sarcoïdose au cours d'un traitement par ipilimumab. Ann Dermatol Venereol. 2012;139(12):B261-2.

9 Kim C, Gao J, Shannon VR, Siefker-Radtke A. Systemic sarcoidosis first manifesting in a tattoo in the setting of immune checkpoint inhibition. BMJ Case Rep. 2016 Oct;2016:bcr2016216217.

10 Reinhard R, Gebhardt C, Schmieder A, Umansky V, Utikal J. Recurrent tattoo reactions in a patient treated with BRAF and MEK inhibitors. J Eur Acad Dermatol Venereol. 2017 Aug;31(8):e375-7.

11 Malissen N, Magis Q, Macagno N, Monsetier S, Troin L, Mallet S, et al. Réactions granulomateuses sur tatouage sous anti-BRAF + anti-MEK révélatrices de fausses progressions. Ann Dermatol Vénéréol. 2017; 144(12):S128-9.

12 Rohmer E, Scrivener JN, Schissler C, Cribier B, Lenormand C. Hypersensibilité retardée aux tatouages induite par un traitement combiné anti-BRAF-anti-MEK. Ann Dermatol Vénéréol. 2017;144(12):s320.

13 Laske J, Meier F, Bauer A, Beissert S, Garzarolli M. Tattoo-associated complications of metastatic melanoma treated with dabrafenib and trametinib. Melanoma Res. 2018 Oct;28(5):485-7.

14 Pileri A, Starace M, Leuzzi M, Agostinelli C, Sabattini E, Morigi A, et al. Granulomatous tattoo reaction in a nivolumab-treated patient. G Ital Dermatol Venereol. 2018 Jun. DOI: 10.23736/S0392-0488.18.06084-4.

15 Rousseau PM, Raimbourg J, Robert M, Dansette D, Dréno B, Peuvrel L; supported by GESTIM Nantes group of cutaneous adverse events induced by cancer treatments. First case of cutaneous sarcoidosis within tattoos under durvalumab. Int J Dermatol. 2019 Jun 4. Epub ahead of print.

16 Giet G, Lebas E, Rorive A, Arrese JE, Nikkels AF. Granulomatous Reactions from Tattoos Following BRAF Inhibitor Therapy. Case Rep Dermatol. 2019 Apr;11(1):101-7.

17 Lheure C, Kramkimel N, Franck N, Laurent-Roussel S, Carlotti A, Queant A, et al. Sarcoidosis in Patients Treated with Vemurafenib for Metastatic Melanoma: A Paradoxical Autoimmune Activation. Dermatology. 2015;231(4):378-84.

18 Sepehri M, Hutton Carlsen K, Serup J. Papulo-Nodular Reactions in Black Tattoos as Markers of Sarcoidosis: Study of 92 Tattoo Reactions from a Hospital Material. Dermatology. 2016;232(6):679-86.

19 Kluger N. Cutaneous Complications Related to Tattoos: 31 Cases from Finland. Dermatology. 2017;233(1):100-9.

20 Amoura A, Haroche J, Emile JF, Barete S, Helias-Rodzewicz Z, Charlotte $\mathrm{F}$, et al. Sarcoidosis occurring during BRAF/MEK inhibitors is associated with paradoxical ERK activation in Erdheim-Chester patients. J Eur Acad Dermatol Venereol. 2019 May 14. Epub ahead of print.

21 Conciatori F, Ciuffreda L, Bazzichetto C, Falcone I, Pilotto S, Bria E, et al. mTOR Cross-Talk in Cancer and Potential for Combination Therapy. Cancers (Basel). 2018 Jan;10(1):E23.

22 Linke M, Pham HT, Katholnig K, Schnöller T, Miller A, Demel F, et al. Chronic signaling via the metabolic checkpoint kinase mTORC1 induces macrophage granuloma formation and marks sarcoidosis progression. Nat Immunol. 2017 Mar;18(3):293-302. 